\title{
Incidence of unexpected red blood cell antibodies in the north of China
}

\author{
Yu-Shiang Lin ${ }^{1}$, Jhy-Sheng Chang ${ }^{2}$, Yan Qiu ${ }^{3}, \mathrm{Fu}-\mathrm{Hsiung} \mathrm{Su}^{4,5}$, Yin-Chun Lan ${ }^{1}$, Nai-Chung Lee , \\ Joyces Wong Lee Mei ${ }^{1}$, Daowang Fan $^{3}$, Tianhong Miao ${ }^{3, *}$ \\ ${ }^{1}$ Department of Clinical Medicine, School of Basic Medical Sciences, Peking University Health Science Center;Beijing, 100191 China. \\ ${ }^{2}$ Department of Laboratory Medicine, National Taiwan University Hospital;Taipei City, 10002 Taiwan \\ ${ }^{3}$ Blood Group Lab, Beijing Red Cross Blood Center; Beijing, 100088 China \\ ${ }^{4}$ Department of Family Medicine, School of Medicine, College of Medicine, Taipei Medical University, Taipei City, 11042 Taiwan. \\ ${ }^{5}$ School of Medicine, Flinders University, Bedford Park, South Australia, 5042 Australia.
}

\begin{abstract}
This study aimed to investigate the frequency of unexpected antibodies and evaluate the cumulative incidence of additional unexpected antibodies in Beijing. From January 1, 2011 to December 31, 2014, blood samples from 2,095 patients from 98 medical institutes in Beijing were sent to the Beijing Red Cross Blood Center for antibody identification. Of the unexpected antibodies, $29.5 \%$ were autoantibodies and $70.5 \%$ were alloantibodies. Anti-E was the most prevalent form of allo-antibodies $(n=445)$, accounting for $52.9 \%$ of the Rh system, followed by anti-M (76.6\% of the MNS system) and then 142 cases of anti-C,e, 128 cases of anti-E,c, and 113 cases of anti$\mathrm{Le}^{\mathrm{a}}$. The cumulative incidences of additional antibodies were $0.55 \%$ (after the first transfusion), $1.82 \%$ (second time), $2.33 \%$ (fourth time), $3.07 \%$ (firth time), and 4.24\% (seventh time). Antibody against the Rh system was the most prevalent, followed by antibodies against MNS, Lewis, Kidd, P1, and Duffy.
\end{abstract}

Keywords: incidence, alloantibody, alloimmunization, autoantibody

\section{INTRODUCTION}

Red blood cell (RBC) unexpected antibodies, including types of autoantibody and alloantibody, is an important and common issue regarding blood transfusion. Antibodies against high-frequency antigen, multiple antibody, and additional unexpected antibodies can challenge the efficacy and safety of blood transfusion ${ }^{[1]}$. Factors that can induce the occurrence of unexpected antibodies include age, history of pregnancy, history of blood transfusion, human leukocyte antigen (HLA)-DR typing, treatment, immune status, and especially, RBC density among people of dif-

*Correspondence to: Tianhong Miao, MS, Chief Medical Technologist, Director of Blood Group Lab, Beijing Red Cross Blood Center, No 37, Beisanhuan, Zhonglu, Beijing, China. TEL: 86-10-8280-7272, FAX: ferent ethnical groups ${ }^{[2-4]}$. For example, anti-Fy ${ }^{\mathrm{a}}$ and anti-D are common among Caucasians, but their corresponding antigens have a positivity rate higher than 99\% among Asians. The Miltenberger antigen is a very low-frequency antigen in the Caucasian populations, while anti- $\mathrm{Mi}^{\mathrm{a}}$ is one of the most common unexpected antibodies among Asians ${ }^{[5,6]}$. Although the prevalence of RBC alloantibody has been reported in China, large-scale data are still lacking because of the difficulties in observing low-frequency RBC and multiple antibodies ${ }^{[7-12]}$.

So far, studies on the cumulative incidence of additional unexpected antibodies are mainly focused on patients receiving multiple transfusions, such as those

86-10-8207-9886, EMAIL: 1448639963@qq.com.

The authors declared that they do not have anything to disclose regarding funding or conflict of interest with respect to this manuscript. 
with diseases and illnesses related to hematologic oncology, hematology, and hemodialysis and organ transplantation ${ }^{[13-16]}$. According to a large-scale research study, the cumulative incidence of additional unexpected antibodies among patients receiving continuous transfusion was $1 \%$ after the transfusion of 5 Units of RBCs, $2.4 \%$ after the transfusion of 10 Units of RBCs, $3.4 \%$ after the transfusion of 20 Units of RBCs, and $6.5 \%$ after the transfusion of 40 Units of $\mathrm{RBCs}^{[17]}$. By far, only one study is available that focused on European populations over a long-term observation period. The study indicated that up to $57 \%$ of patients diagnosed with unexpected antibody had additional antibodies (median amount of transfused RBCs, 2 Units) ${ }^{[18]}$. So far, no such a large-scale research that focuses on Asian ethnical populations has been reported.

The Beijing Red Cross Blood Center (BRCBC) conducts tests for antibody identification and transfusion blood cross-matching for patients with RBC alloantibody diseases, and samples are obtained from all hospitals in Beijing region. By statistically analyzing the results from the tests conducted between 2011 and 2014, this study aimed to observe the prevalence of unexpected antibodies among Asians in the Beijing region and the cumulative incidence of additional antibodies among patients diagnosed with unexpected antibody in the course of continuous transfusion of RBCs, based on the frequency and number of transfusions.

\section{MATERIALS AND METHODS}

\section{Sample collection}

This study collected EDTA test-tube samples sent to the Blood Group Laboratory (BGL) of the BRCBC from 98 medical institutions in Beijing between January 1, 2011 and December 31, 2014. The number of patients with unexpected antibodies was 2,095 , with 7,719 records, including antibody identification and cross-matching test. All the samples were collected after the pre-transfusion blood test at the hospitals to exclude samples from donors who had positive unexpected antibody. Before conducting the clinical blood transfusion, unexpected antibody screening was needed in the hospitals in Beijing. And then crossmatching would be performed at each hospital if the result was negative. Samples with positive screening results were sent to the BRCBC for unexpected antibody identification. The BRCBC was responsible for all the following tests for antigen compatible blood component screening and cross-matching. For each sample received, the BRCBC first performed $\mathrm{ABO}$ Rh typing and repeated unexpected antibody screening by conventional tube techniques in room temperature and gel column test simultaneously. A gel column test was performed for antibody identification to screen for antibodies. Only a few individual samples that showed negative results in the gel column test were identified positive in the room temperature tube test for antibody identification. The other procedures such as phenotyping, sample reception, report documentation, and report writing were all based on regulations and the standard procedures specified by the BGL of the BRCBC.

\section{Antibody screening and identification}

Screened and identified cells used at the BGL of the BRCBC cover antigen systems, including Rh, MNS, Duffy, Kidd, Kell, Lewis, Lewis, Pl, Xg, and Lutheran, as well as low-frequency antigens such as $\mathrm{Di}^{\mathrm{a}}$ and Mur (Bio-RAD and Sanquin), which have relatively high prevalence in Asia. Detection methods include conventional tube techniques in room temperature and gel column test (Bio-Rad).

For the calculation of cumulative incidence, only the records of patients who received transfusion more than twice at an interval of more than 3 days were included in the analysis. The exclusion criteria were as follows: 1) patients who only had one blood transfusion record; and 2) infants younger than half of one year, considering the interference of unexpected antibody from the mothers. The BRCBC took charge of the identification of all the unexpected antibodies, the antigen compatible blood component test, and the cross-matching of the positive antibody in Beijing region. Additional antibody is defined as unexpected antibody that has been diagnosed by the Blood Group Room and has more than two records of transfusion. Unmatched cross-matching results between the initial blood test and the following pre-transfusion blood test and the new unexpected antibody were obtained after requalification. The end point was the last transfusion record taken by the BRCBC. The frequency and amount of transfusion were assessed along the time axes.

\section{Statistical analysis}

The prevalence of unexpected and primary antibodies has been defined as first-time specific unexpected antibody diagnosed by the BRCBC. The cumulative incidence of additional alloantibodies was estimated by using the Kaplan-Meier table and for observing possible factors with additional alloantibodies. We stratified the Kaplan-Meier survival tables according to sex and age. 
A chi-square and Fisher exact tests were used to evaluate comparisons between the study groups. Comparison results with a $2-$ sided $P$ value $<0.05$ were considered statistically significant. All the statistical analyses were conducted by using SPSS version 19.0 (SPSS Inc., Chicago, IL, USA).

\section{RESULTS}

\section{Prevalence of $\mathbf{R B C}$ antibody}

Among the 2,095 patients with first-time diagnose of specific unexpected antibodies by the BRCBC, 617 had autoantibodies (29.5\%) and 1,478 had RBC alloantibodies $(70.5 \%)$. By using the sixth China Census in 2010 that surveyed 1.3 billion populations as reference, the effects of age and sex on the occurrence of unexpected antibody were both statistically significant. Being elderly and female were risk factors of unexpected antibodies occurrence. Among all the types of alloantibody, antibody against single systems was the most prevalent form (95.8\%), followed by antibody against $\mathrm{Rh}(59.4 \%)$ and antibodies against MNS (25.9\%) and Lewis (9.0\%). Antibodies against multiple systems only account for $4.5 \%$ of the cases, of which antibody against Rh with Kidd was the most prevalent $(50.0 \%)$, followed by antibody against $\mathrm{Rh}$ with MNS (31.7\%) and antibody against Rh with Duffy (8.3\%; Table 1).
Anti-E was the most prevalent form of antibody against single systems, with 445 cases in the Rh system $(52.9 \%)$, followed by anti-M with 281 cases in the MNS system (76.6\%), 142 cases of anti-C,e, 128 cases of anti-E,c, and 113 cases of anti-Lea (Table 2). Anti-Jka with anti-E and anti-Jka with anti-c,E were the most prevalent forms among antibodies against multiple systems (Table 3 ).

\section{Cumulative incidence of additional RBC al- loantibody}

Among the 2,095 patients with positive antibody results, after exclusion of 1,539 patients with only one transfusion record or without tracking method and 5 patients younger than 0.5 year, 551 patients were included in the cumulative incidence study (Table 4). Among the 551 patients, 538 did not have additional antibodies during the tracking period (control group) and 13 had additional antibodies (case group). In total, 2,515 tracking records were collected (control: 2,477 Vs. case: 38 ) and 14,124 units of RBC blood component was transfused (control: 14,056 vs. case: 68 ). The case group consisted only 2 males ( $15.4 \%$ ), and the male percentage was much lower than the female percentage in the control group $(P=0.043)$. Other information is shown in Table 4. The cumulative incidence of additional antibodies was $0.55 \%$ at the first time, $1.82 \%$ at the second time, $2.33 \%$ at the fourth

Table 1 Frequencies of the 2,095 patients with autoantibodies and alloantibodies

\begin{tabular}{|c|c|c|c|c|c|c|c|c|c|c|c|c|c|c|}
\hline & \multicolumn{7}{|c|}{ Gender } & \multicolumn{6}{|c|}{ Age } & \multirow[b]{2}{*}{$P$} \\
\hline & Number & $\%$ & Fema & le, $n(\%)$ & Mal & $n(\%)$ & $P$ & $<1$ & $n(\%)$ & $18-6$ & $5, n(\%)$ & $>65$ & $n(\%)$ & \\
\hline Unexpected antibodies in total & 2,095 & & 1,336 & & 759 & & $<0.001$ & 133 & & 1,285 & & 677 & & $<0.001$ \\
\hline Specific allo-antibodies & 1,478 & $70.5 \%$ & 946 & $64.0 \%$ & 532 & $36.0 \%$ & $<0.001$ & 77 & $5.2 \%$ & 963 & $65.2 \%$ & 438 & $29.6 \%$ & $<0.001$ \\
\hline Single system & 1,416 & $95.8 \%$ & 905 & $63.9 \%$ & 511 & $36.1 \%$ & $<0.001$ & 76 & $5.4 \%$ & 919 & $64.9 \%$ & 421 & $29.7 \%$ & $<0.001$ \\
\hline MNS & 367 & $25.9 \%$ & 222 & $60.5 \%$ & 145 & $39.5 \%$ & & 41 & $11.2 \%$ & 226 & $61.6 \%$ & 100 & $27.2 \%$ & \\
\hline Lewis & 127 & $9.0 \%$ & 89 & $70.1 \%$ & 38 & $29.9 \%$ & & 7 & $5.5 \%$ & 103 & $81.1 \%$ & 17 & $13.4 \%$ & \\
\hline Kidd & 56 & $4.0 \%$ & 35 & $62.5 \%$ & 21 & $37.5 \%$ & & 1 & $1.8 \%$ & 37 & $66.1 \%$ & 18 & $32.1 \%$ & \\
\hline Duffy & 7 & $0.5 \%$ & 4 & $57.1 \%$ & 3 & $42.9 \%$ & & 0 & $0.0 \%$ & 7 & $100.0 \%$ & 0 & $0.0 \%$ & \\
\hline $\mathrm{Pl}$ & 12 & $0.8 \%$ & 7 & $58.3 \%$ & 5 & $41.7 \%$ & & 1 & $8.3 \%$ & 9 & $75.0 \%$ & 2 & $16.7 \%$ & \\
\hline Lutheran & 2 & $0.1 \%$ & 1 & $50.0 \%$ & 1 & $50.0 \%$ & & 0 & $0.0 \%$ & 1 & $50.0 \%$ & 1 & $50.0 \%$ & \\
\hline Kell & 3 & $0.2 \%$ & 1 & $33.3 \%$ & 2 & $66.7 \%$ & & 0 & $0.0 \%$ & 2 & $66.7 \%$ & 1 & $33.3 \%$ & \\
\hline $\mathrm{Xg}$ & 1 & $0.1 \%$ & 1 & $100.0 \%$ & 0 & $0.0 \%$ & & 0 & $0.0 \%$ & 1 & $100.0 \%$ & 0 & $0.0 \%$ & \\
\hline Double system & 60 & $4.1 \%$ & 40 & $66.7 \%$ & 20 & $33.3 \%$ & $<0.001$ & 1 & $1.7 \%$ & 42 & $70.0 \%$ & 17 & $28.3 \%$ & $<0.001$ \\
\hline $\mathrm{Rh}+\mathrm{MNS}$ & 19 & $31.7 \%$ & 12 & $63.2 \%$ & 7 & $36.8 \%$ & & 0 & $0.0 \%$ & 14 & $73.7 \%$ & 5 & $26.3 \%$ & \\
\hline $\mathrm{Rh}+$ Lewis & 2 & $3.3 \%$ & 1 & $50.0 \%$ & 1 & $50.0 \%$ & & 0 & $0.0 \%$ & 2 & $100.0 \%$ & 0 & $0.0 \%$ & \\
\hline $\mathrm{Rh}+\mathrm{Kidd}$ & 30 & $50.0 \%$ & 18 & $60.0 \%$ & 12 & $40.0 \%$ & & 1 & $3.3 \%$ & 20 & $66.7 \%$ & 9 & $30.0 \%$ & \\
\hline Rh + Duffy & 5 & $8.3 \%$ & 3 & $60.0 \%$ & 2 & $40.0 \%$ & & 0 & $0.0 \%$ & 5 & $100.0 \%$ & 0 & $0.0 \%$ & \\
\hline $\mathrm{Rh}+$ Diego & 3 & $5.0 \%$ & 1 & $33.3 \%$ & 2 & $66.7 \%$ & & 0 & $0.0 \%$ & 0 & $0.0 \%$ & 3 & $100.0 \%$ & \\
\hline MNS + Lewis & 1 & $1.7 \%$ & 1 & $100.0 \%$ & 0 & $0.0 \%$ & & 0 & $0.0 \%$ & 1 & $100.0 \%$ & 0 & $0.0 \%$ & \\
\hline Trible system & 2 & $0.1 \%$ & 1 & $50.0 \%$ & 1 & $50.0 \%$ & & 0 & $0.0 \%$ & 2 & $100.0 \%$ & 0 & $0.0 \%$ & \\
\hline Rh + Kidd + Duffy & 2 & $100.0 \%$ & 1 & $50.0 \%$ & 1 & $50.0 \%$ & & 0 & $0.0 \%$ & 2 & $100.0 \%$ & 0 & $0.0 \%$ & \\
\hline
\end{tabular}

Specific allo-antibodies with or without autoantibody 
Table 2 Frequencies of alloantibody against single RBC system

\begin{tabular}{|c|c|c|c|c|c|c|c|c|c|c|c|c|}
\hline \multirow[b]{3}{*}{$\mathbf{R h}$} & \multirow[b]{2}{*}{ Number } & \multirow[b]{2}{*}{$\%$} & \multicolumn{4}{|c|}{ Gender } & \multicolumn{6}{|c|}{ Age } \\
\hline & & & \multicolumn{2}{|c|}{ Female, $n(\%)$} & \multicolumn{2}{|c|}{ Male, $n(\%)$} & \multicolumn{2}{|c|}{$<18, n(\%)$} & \multicolumn{2}{|c|}{$18-65, n(\%)$} & \multicolumn{2}{|c|}{$>65, n(\%)$} \\
\hline & 841 & & 545 & & 296 & & 24 & & 535 & & 280 & \\
\hline Anti-C & 27 & $3.2 \%$ & 15 & $55.6 \%$ & 12 & $44.4 \%$ & 2 & $7.4 \%$ & 17 & $63.0 \%$ & 8 & $29.6 \%$ \\
\hline Anti-c & 4 & $0.5 \%$ & 1 & $25.0 \%$ & 3 & $75.0 \%$ & 1 & $25.0 \%$ & 0 & $0.0 \%$ & 3 & $75.0 \%$ \\
\hline Anti-D & 65 & $7.7 \%$ & 44 & $67.7 \%$ & 21 & $32.3 \%$ & 2 & $3.1 \%$ & 45 & $69.2 \%$ & 18 & $27.7 \%$ \\
\hline Anti-E & 445 & $52.9 \%$ & 306 & $68.8 \%$ & 139 & $31.2 \%$ & 12 & $2.7 \%$ & 284 & $63.8 \%$ & 149 & $33.5 \%$ \\
\hline Anti-e & 5 & $0.6 \%$ & 2 & $40.0 \%$ & 3 & $60.0 \%$ & 0 & $0.0 \%$ & 4 & $80.0 \%$ & 1 & $20.0 \%$ \\
\hline Anti-F & 2 & $0.2 \%$ & 2 & $100.0 \%$ & 0 & $0.0 \%$ & 0 & $0.0 \%$ & 0 & $0.0 \%$ & 2 & $100.0 \%$ \\
\hline Anti-Hr0 & 2 & $0.2 \%$ & 2 & $100.0 \%$ & 0 & $0.0 \%$ & 0 & $0.0 \%$ & 1 & $50.0 \%$ & 1 & $50.0 \%$ \\
\hline Anti-C,e & 142 & $16.9 \%$ & 79 & $55.6 \%$ & 63 & $44.4 \%$ & 4 & $2.8 \%$ & 83 & $58.5 \%$ & 55 & $38.7 \%$ \\
\hline Anti-E,c & 128 & $15.2 \%$ & 80 & $62.5 \%$ & 48 & $37.5 \%$ & 3 & $2.3 \%$ & 90 & $70.3 \%$ & 35 & $27.3 \%$ \\
\hline Anti-D,C & 14 & $1.7 \%$ & 10 & $71.4 \%$ & 4 & $28.6 \%$ & 0 & $0.0 \%$ & 7 & $50.0 \%$ & 7 & $50.0 \%$ \\
\hline Anti-D,E & 7 & $0.8 \%$ & 2 & $28.6 \%$ & 5 & $71.4 \%$ & 2 & $28.6 \%$ & 2 & $28.6 \%$ & 3 & $42.9 \%$ \\
\hline MNS & 367 & & 222 & & 145 & & 41 & & 226 & & 100 & \\
\hline Anti-M & 281 & $76.6 \%$ & 165 & $58.7 \%$ & 116 & $41.3 \%$ & 39 & $13.9 \%$ & 171 & $60.9 \%$ & 71 & $25.3 \%$ \\
\hline Anti-N & 4 & $1.1 \%$ & 3 & $75.0 \%$ & 1 & $25.0 \%$ & 0 & $0.0 \%$ & 2 & $50.0 \%$ & 2 & $50.0 \%$ \\
\hline Anti-S & 29 & $7.9 \%$ & 23 & $79.3 \%$ & 6 & $20.7 \%$ & 0 & $0.0 \%$ & 24 & $82.8 \%$ & 5 & $17.2 \%$ \\
\hline Anti-"Mi" & 53 & $14.4 \%$ & 31 & $58.5 \%$ & 22 & $41.5 \%$ & 2 & $3.8 \%$ & 29 & $54.7 \%$ & 22 & $41.5 \%$ \\
\hline Lewis & 127 & & 89 & & 38 & & 7 & & 103 & & 17 & \\
\hline Anti-Le & 113 & $89.0 \%$ & 78 & $69.0 \%$ & 35 & $31.0 \%$ & 6 & $5.3 \%$ & 93 & $82.3 \%$ & 14 & $12.4 \%$ \\
\hline Anti-Le ${ }^{b}$ & 13 & $10.2 \%$ & 10 & $76.9 \%$ & 3 & $23.1 \%$ & 1 & $7.7 \%$ & 10 & $76.9 \%$ & 2 & $15.4 \%$ \\
\hline Anti-Le $^{\mathrm{a}},-\mathrm{Le}^{\mathrm{b}}$ & 1 & $0.8 \%$ & 1 & $100.0 \%$ & 0 & $0.0 \%$ & 0 & $0.0 \%$ & 0 & $0.0 \%$ & 1 & $100.0 \%$ \\
\hline Kidd & 56 & & 35 & & 21 & & 1 & & 37 & & 18 & \\
\hline Anti-Jk ${ }^{\mathrm{a}}$ & 19 & $33.9 \%$ & 11 & $57.9 \%$ & 8 & $42.1 \%$ & 0 & $0.0 \%$ & 15 & $78.9 \%$ & 4 & $21.1 \%$ \\
\hline Anti-Jk ${ }^{b}$ & 30 & $53.6 \%$ & 20 & $66.7 \%$ & 10 & $33.3 \%$ & 1 & $3.3 \%$ & 16 & $53.3 \%$ & 13 & $43.3 \%$ \\
\hline Anti-Jk ${ }^{3}$ & 7 & $12.5 \%$ & 4 & $57.1 \%$ & 3 & $42.9 \%$ & 0 & $0.0 \%$ & 6 & $85.7 \%$ & 1 & $14.3 \%$ \\
\hline Duffy & 7 & & 4 & & 3 & & 0 & & 7 & & 0 & \\
\hline Anti-Fy $^{\mathrm{a}}$ & 4 & $57.1 \%$ & 2 & $50.0 \%$ & 2 & $50.0 \%$ & 0 & $0.0 \%$ & 4 & $100.0 \%$ & 0 & $0.0 \%$ \\
\hline Anti-Fy ${ }^{b}$ & 3 & $42.9 \%$ & 2 & $66.7 \%$ & 1 & $33.3 \%$ & 0 & $0.0 \%$ & 3 & $100.0 \%$ & 0 & $0.0 \%$ \\
\hline P1 & 12 & & 7 & & 5 & & 1 & & 9 & & 2 & \\
\hline Anti-P1 & 12 & $100.0 \%$ & 7 & $58.3 \%$ & 5 & $41.7 \%$ & 1 & $8.3 \%$ & 9 & $75.0 \%$ & 2 & $16.7 \%$ \\
\hline Lutheran & 2 & & 1 & & 1 & & 0 & & 1 & & 1 & \\
\hline Anti-Lua & 2 & $100.0 \%$ & 1 & $50.0 \%$ & 1 & $50.0 \%$ & 0 & $0.0 \%$ & 1 & $50.0 \%$ & 1 & $50.0 \%$ \\
\hline Kell & 3 & & 1 & & 2 & & 0 & & 2 & & 1 & \\
\hline Anti-K & 3 & $100.0 \%$ & 1 & $33.3 \%$ & 2 & $66.7 \%$ & 0 & $0.0 \%$ & 2 & $66.7 \%$ & 1 & $33.3 \%$ \\
\hline Xg & 1 & & 1 & & 0 & & 0 & & 1 & & 0 & \\
\hline Anti-Xg & 1 & $100.0 \%$ & 1 & $100.0 \%$ & 0 & $0.00 \%$ & 0 & $0.00 \%$ & 1 & $100.00 \%$ & 0 & $0.00 \%$ \\
\hline
\end{tabular}

Numbers under the \% column represent the percentage of individual antibody in each system.

Numbers in horizontal rows under the gender and age column represent the percentage of individual group in respective columns.

time, $3.07 \%$ at the fifth time, and $4.24 \%$ at the seventh time. The cumulative incidence rates of the number of RBC units, from 2 to 14 units (at 2-unit intervals), were $0.55 \%, 1.31 \%, 1.78 \%, 2.10 \%, 2.52 \%, 3.05 \%$, and $3.73 \%$, respectively (Table 4).

As both age and sex had significant differences, we used the Kaplan-Meier survival curves for the analysis of sex-specific cumulative incidence of blood transfusion. The cumulative incidence of additional antibodies was significantly higher among females than that among males (log rank test: 0.035 and 0.038 , respectively), from the perspective of either transfusion frequency or number. The effect of age on the cumulative incidence was not statistically significant (Fig. 1,Table 5).

Additional antibodies had 7 types of unexpected antibodies, including 8 cases of antibody against $\mathrm{Rh}$

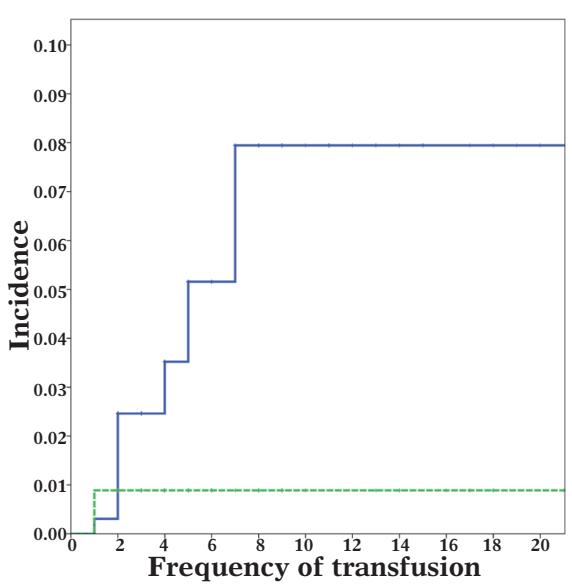

Fig. 1. Gender- specific cumulative incidence in immunized patients according to frequency of transfused subjects.

Solid line represents women and dotted line represents men. 
Table 3 Frequencies of alloantibody against Multiple RBC system

\begin{tabular}{|c|c|c|c|c|c|c|c|c|c|c|c|c|}
\hline \multirow[b]{3}{*}{ Double system } & \multirow[b]{2}{*}{ Number } & \multirow[b]{2}{*}{ Constituent Rate } & \multicolumn{4}{|c|}{ Gender } & \multicolumn{6}{|c|}{ Age } \\
\hline & & & \multicolumn{2}{|c|}{ Female, $n(\%)$} & \multicolumn{2}{|c|}{ Male, $n(\%)$} & \multicolumn{2}{|c|}{$<18, n(\%)$} & \multicolumn{2}{|c|}{$18-65, n(\%)$} & \multicolumn{2}{|c|}{$>65, n(\%)$} \\
\hline & & & & & & & & & & & & \\
\hline Rh + MNS & 19 & & 12 & & 7 & & $\mathbf{0}$ & & 14 & & 5 & \\
\hline Anti-M+anti-C & 1 & $5.3 \%$ & 1 & $100.0 \%$ & 0 & $0.0 \%$ & 0 & $0.0 \%$ & 1 & $100.0 \%$ & 0 & $0.0 \%$ \\
\hline Anti-M+anti-E & 4 & $21.1 \%$ & 3 & $75.0 \%$ & 1 & $25.0 \%$ & 0 & $0.0 \%$ & 4 & $100.0 \%$ & 0 & $0.0 \%$ \\
\hline Anti-N+anti-E & 1 & $5.3 \%$ & 0 & $0.0 \%$ & 1 & $100.0 \%$ & 0 & $0.0 \%$ & 0 & $0.0 \%$ & 1 & $100.0 \%$ \\
\hline Anti-S+anti-C & 1 & $5.3 \%$ & 1 & $100.0 \%$ & 0 & $0.0 \%$ & 0 & $0.0 \%$ & 0 & $0.0 \%$ & 1 & $100.0 \%$ \\
\hline Anti-S+anti-E & 3 & $15.8 \%$ & 2 & $66.7 \%$ & 1 & $33.3 \%$ & 0 & $0.0 \%$ & 3 & $100.0 \%$ & 0 & $0.0 \%$ \\
\hline Anti-Mia+anti-E & 2 & $10.5 \%$ & 1 & $50.0 \%$ & 1 & $50.0 \%$ & 0 & $0.0 \%$ & 2 & $100.0 \%$ & 0 & $0.0 \%$ \\
\hline Anti-M+anti-c,-E & 4 & $21.1 \%$ & 3 & $75.0 \%$ & 1 & $25.0 \%$ & 0 & $0.0 \%$ & 3 & $75.0 \%$ & 1 & $25.0 \%$ \\
\hline Anti-Mia+anti-c,-E & 1 & $5.3 \%$ & 0 & $0.0 \%$ & 1 & $100.0 \%$ & 0 & $0.0 \%$ & 0 & $0.0 \%$ & 1 & $100.0 \%$ \\
\hline Anti-N+anti-C,-e & 1 & $5.3 \%$ & 1 & $100.0 \%$ & 0 & $0.0 \%$ & 0 & $0.0 \%$ & 1 & $100.0 \%$ & 0 & $0.0 \%$ \\
\hline Anti-S+anti-C,-e & 1 & $5.3 \%$ & 0 & $0.0 \%$ & 1 & $100.0 \%$ & 0 & $0.0 \%$ & 0 & $0.0 \%$ & 1 & $100.0 \%$ \\
\hline Rh + Lewis & 2 & & 1 & & 1 & & $\mathbf{0}$ & & 2 & & 0 & \\
\hline Anti-Le + anti-D & 1 & $50.0 \%$ & 1 & $100.0 \%$ & 0 & $0.0 \%$ & 0 & $0.0 \%$ & 1 & $100.0 \%$ & 0 & $0.0 \%$ \\
\hline Anti-Le ${ }^{a}+a n t i-E$ & 1 & $50.0 \%$ & 0 & $0.0 \%$ & 1 & $100.0 \%$ & 0 & $0.0 \%$ & 1 & $100.0 \%$ & 0 & $0.0 \%$ \\
\hline Rh + Kidd & 30 & & 18 & & 12 & & 1 & & 20 & & 9 & \\
\hline Anti-Jk ${ }^{\mathrm{a}}+$ anti-C & 2 & $6.7 \%$ & 1 & $50.0 \%$ & 1 & $50.0 \%$ & 0 & $0.0 \%$ & 1 & $50.0 \%$ & 1 & $50.0 \%$ \\
\hline Anti-Jk ${ }^{\mathrm{a}}+$ anti-E & 9 & $30.0 \%$ & 5 & $55.6 \%$ & 4 & $44.4 \%$ & 0 & $0.0 \%$ & 7 & $77.8 \%$ & 2 & $22.2 \%$ \\
\hline Anti-Jk $\mathrm{k}^{\mathrm{b}}+$ anti-E & 5 & $16.7 \%$ & 5 & $100.0 \%$ & 0 & $0.0 \%$ & 0 & $0.0 \%$ & 3 & $60.0 \%$ & 2 & $40.0 \%$ \\
\hline Anti-Jk ${ }^{\mathrm{a}}+$ anti-c, $-\mathrm{E}$ & 6 & $20.0 \%$ & 3 & $50.0 \%$ & 3 & $50.0 \%$ & 0 & $0.0 \%$ & 3 & $50.0 \%$ & 3 & $50.0 \%$ \\
\hline Anti-Jk ${ }^{\mathrm{b}}+$ anti-c,-E & 4 & $13.3 \%$ & 1 & $25.0 \%$ & 3 & $75.0 \%$ & 1 & $25.0 \%$ & 3 & $75.0 \%$ & 0 & $0.0 \%$ \\
\hline Anti-Jk ${ }^{\mathrm{a}}+$ anti-C,-e & 2 & $6.7 \%$ & 2 & $100.0 \%$ & 0 & $0.0 \%$ & 0 & $0.0 \%$ & 2 & $100.0 \%$ & 0 & $0.0 \%$ \\
\hline Anti-Jk ${ }^{\mathrm{b}}+$ anti-C,-e & 1 & $3.3 \%$ & 1 & $100.0 \%$ & 0 & $0.0 \%$ & 0 & $0.0 \%$ & 1 & $100.0 \%$ & 0 & $0.0 \%$ \\
\hline Anti-Jk + anti-D,-E & 1 & $3.3 \%$ & 0 & $0.0 \%$ & 1 & $100.0 \%$ & 0 & $0.0 \%$ & 0 & $0.0 \%$ & 1 & $100.0 \%$ \\
\hline Rh + Duffy & 5 & & 3 & & 2 & & 0 & & 5 & & 0 & \\
\hline Anti-Fy ${ }^{b}+$ anti-E & 5 & $100.0 \%$ & 3 & $60.0 \%$ & 2 & $40.0 \%$ & 0 & $0.0 \%$ & 5 & $100.0 \%$ & 0 & $0.0 \%$ \\
\hline Rh + Diego & 3 & & 1 & & 2 & & $\mathbf{0}$ & & 0 & & 3 & \\
\hline Anti-Di ${ }^{\mathrm{a}}+$ anti-C & 2 & $66.7 \%$ & 0 & $0.0 \%$ & 2 & $100.0 \%$ & 0 & $0.0 \%$ & 0 & $0.0 \%$ & 2 & $100.0 \%$ \\
\hline Anti-Di ${ }^{\mathrm{a}}+$ anti-E & 1 & $33.3 \%$ & 1 & & 0 & & 0 & $0.0 \%$ & 0 & $0.0 \%$ & 1 & $100.0 \%$ \\
\hline MNS + Lewis & 1 & & 1 & & 0 & & 0 & & 1 & & 0 & \\
\hline Anti-M+anti-Le ${ }^{\mathrm{a}}$ & 1 & $100.0 \%$ & 1 & $100.0 \%$ & 0 & $0.0 \%$ & 0 & $0.0 \%$ & 1 & $100.0 \%$ & 0 & $0.0 \%$ \\
\hline \multicolumn{13}{|l|}{ Trible system } \\
\hline Rh + Kidd + Duffy & 2 & & 1 & & 1 & & 0 & & 2 & & 0 & \\
\hline 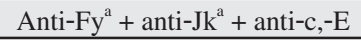 & 2 & $100.0 \%$ & 1 & $50.0 \%$ & 1 & $50.0 \%$ & 0 & $0.0 \%$ & 2 & $100.0 \%$ & 0 & $0.0 \%$ \\
\hline
\end{tabular}

Numbers under the $\%$ column represent the percentage of individual antibody in combined system.

Numbers in horizontal rows under the gender and age column represent the percentage of individual group in respective columns.

Table 4 Baseline to cumulative incidence of immunized patients

\begin{tabular}{lccc}
\hline & Consistency & Additional unexpected & \\
& $(n=538)$ & antibodies $(n=13)$ & $P$ \\
\hline Sex-male(\%) & $228(42.4 \%)$ & $2(15.4 \%)$ & 0.043 \\
Age in years -median( IQR) & $59.0(30.0)$ & $61.0(32.0)$ & 0.783 \\
Cumulative units and frequency & $14,056,2,477$ & 68,38 & \\
Units per patient -median( IQR, Max) & $8(8,178)$ & $4(6,14)$ & 0.006 \\
Follow-up frequency -median( IQR, Max) & $3(2,79)$ & $4(1,7)$ & $<0.001$ \\
\hline
\end{tabular}

Table 5 Cumulative incidence of additional antibody formation with frequency of transfused and RBC units after transfusion in alloimmunized patients.

\begin{tabular}{|c|c|c|c|c|c|c|c|c|c|c|c|}
\hline \multicolumn{6}{|c|}{ Red Blood Cell Unit } & \multicolumn{6}{|c|}{ Transfusion Frequency } \\
\hline & \multicolumn{2}{|c|}{ Sex, $n(\%)$} & \multicolumn{2}{|c|}{ Age, $n(\%)$} & \multirow[b]{2}{*}{ Total } & \multirow[b]{2}{*}{ Number } & \multicolumn{2}{|c|}{ Sex, $n(\%)$} & \multicolumn{2}{|c|}{ Age, $n(\%)$} & \multirow[b]{2}{*}{ Total } \\
\hline Number & Female & Male & $18-65$ & $>65$ & & & Female & Male & $18-65$ & $>65$ & \\
\hline 2 & $0.31 \%$ & $0.89 \%$ & & $1.41 \%$ & $0.55 \%$ & 1 & $0.31 \%$ & $0.89 \%$ & & $1.42 \%$ & $0.55 \%$ \\
\hline 4 & $1.61 \%$ & & $0.66 \%$ & $2.38 \%$ & $1.31 \%$ & 2 & $2.46 \%$ & & $1.61 \%$ & $2.36 \%$ & $1.82 \%$ \\
\hline 6 & $2.45 \%$ & & $1.44 \%$ & & $1.78 \%$ & 4 & $3.52 \%$ & & $2.52 \%$ & & $2.33 \%$ \\
\hline 8 & $3.01 \%$ & & $1.94 \%$ & & $2.10 \%$ & 5 & $5.16 \%$ & & $3.97 \%$ & & $3.07 \%$ \\
\hline 10 & $3.77 \%$ & & & $3.48 \%$ & $2.52 \%$ & 7 & $7.95 \%$ & & & $4.68 \%$ & $4.24 \%$ \\
\hline 12 & $4.84 \%$ & & $2.85 \%$ & & $3.05 \%$ & & & & & & \\
\hline 14 & $6.28 \%$ & & $4.00 \%$ & & $3.73 \%$ & & & & & & \\
\hline
\end{tabular}


Table 6 Disease and diversification of irregular Abs at first and after transfusions in immunized patients

\begin{tabular}{lcc}
\hline $\begin{array}{l}\text { Consistent Unexpected Abs } \\
\text { Type }\end{array}$ & Additional immunization & Disease \\
\hline Auto $\mathrm{Ab}$ & Additional Abs & Gastrointestinal bleeding \\
& Auto Ab+anti-Ce & Systemic lupus erythematosus \\
& Auto Ab+anti-E*2 & Evans syndrome \\
Anti-cE & Auto Ab+anti-Ce & Anemia \\
& Anti-Kp+anti-E & Myelodysplastic syndrome \\
& Auto Ab & Cervical cancer \\
Non-specific $\mathrm{Ab}$ & Anti-E & Chronic Kidney Disease with liver cirrhosis \\
& Auto Ab+anti-Ce & Urinary bladder cancer \\
Anti-M & Auto Ab & Gastrointestinal bleeding \\
Anti-E & Anti-M+anti-cE & Rectal cancer \\
Anti-Fy & Anti-E+anti-S & Acute myelocytic leukemia \\
Anti-D+Anti-Le ${ }^{\mathrm{a}}$ & Anti-Fy + anti-Jk & Autoimmune cirrhosis \\
\hline
\end{tabular}

system (62\%) and 2 cases of autoantibody (15.4\%), as well as anti-Kp ${ }^{\mathrm{a}}$, anti-S, and anti-Jk ${ }^{\mathrm{b}}(7.6 \%$ each, 1 case per type). The disease and antibody diversification of the 13 cases of additional antibodies is summarized in Table 6.

\section{Discussion}

This is a large-scale report of patients with positive unexpected antibodies from 98 medical institutes in Beijing during 2011-2014. With reference to previous studies on immunized patients with unexpected antibodies in China and converting the denominator to fit our study, our study is estimated to cover to 580,000 patients who underwent antibody screening and a total population of 5.37 million $^{[9-12]}$. Taking the data from the China Census in 2010 as reference, we found that being elderly and female are risk factors that led to the relatively high occurrence of unexpected antibodies. Our statistical analysis encompassed relatively common unexpected antibody types and rare unexpected antibodies. To our knowledge, this study is a largescale study that is highly diversified and based on a large-scale sample analysis in China.

Antibodies against the MNS and Lewis systems rank second (25.9\%), and antibody against single systems ranks third (9.0\%), followed by antibody against the Kidd system (4\%), $\mathrm{P}_{1}$ system $(0.8 \%)$, and Duffy system $(0.5 \%)$. As such, we predicted that the co-expression of antibody against double systems and antibody against the Rh system should rank the second, third, or fourth place. However, the order of prevalence of co-expression with antibody against the $\mathrm{Rh}$ system was observed to be as follows: antibody against the Kidd system > antibody against the MNS system $>$ antibody against the Duffy system. The coexpression phenomenon among the antibodies against the Kidd, Duffy, and Rh systems shed light on the op- tion of preventive phenotype matching for the prevention of multiple antibodies in patients with unexpected antibodies.

Anti- $\mathrm{Di}^{\mathrm{a}}$ can only be expressed under the presence of some other antibodies, and anti-Mi ${ }^{\mathrm{a}}$ has a significantly high prevalence rate in Asia. Although screen cells used by BRCBC contain $\mathrm{Mi}^{\mathrm{a}}$ and $\mathrm{Di}^{\mathrm{a}}$ antigens, whether or not to include $\mathrm{Mi}^{\mathrm{a}}$ and $\mathrm{Di}^{\mathrm{a}}$ antigens in screen cells is still controversial ${ }^{[5,6,18-21]}$. Currently, screen cells used by medical institutes in Beijing are from Europe or the United States, and most of which are not $\mathrm{Di}^{\mathrm{a}-}$ or $\mathrm{Mi}^{\mathrm{a}-}$ expressed RBCs. Studies conducted in Thailand and Malaysia showed that the prevalence of anti-Mi ${ }^{\mathrm{a}}$ among unexpected antibodies was no less than that of anti- $\mathrm{E}^{[6,19]}$. There was even a $7.4 \%$ antibody prevalence rate among blood donors in Taiwan, with an antigen positivity rate of about $5.5 \%{ }^{[5]}$. Follow-up observation is necessary to determine whether to recommend the inclusion of anti- $\mathrm{Mi}^{\mathrm{a}}$ in screen cells in China.

In our study, the prevalence of additional antibodies among patients who had the same type of immune disease was only $2.4 \%$, and the cumulative incidence was about $4.24 \%$ for frequency and $3.73 \%$ for number. In addition, the cumulative incidence among females was significantly higher than that among males. Results from our study are different from those obtained by J. G. van der Bom in their study, in which the cumulative incidence was up to $20 \%{ }^{[17]}$. The sample collection method might be the reason for the difference because the previous study was a 20 -year follow-up observation in 2 large hospitals, whereas our study obtained data from all immunized patients in Beijing in the period 2011-2014. Between these two studies, the differences in blood transfusion risk due to medical technologies in different ages, immune diversity and status between different ethnical 
groups, RBC count per unit, and even fertility policies can affect the results. Second, the data used in our study were collected from all hospitals in Beijing and the diseases were relatively complicated and diverse, which are different from the diseases and illnesses studied in medical centers. Significant growths have been observed in China's economy and medical technology since 2000 . Thus, we believe that our study can more closely reflect the transfusion risks induced by the medical technology in Beijing in the past 5 years. Our study showed that the cumulative incidence occurred at the seventh blood transfusion and with 14 $\mathrm{U}$ of transfused RBCs. In addition, it also showed that additional antibody is most likely to occur immediately after the first appearance of antibody. These observations are different from those in foreign studies. Whether the differences are related to immune status such as HLA is still under further investigation ${ }^{[3]}$. Based on the types of additional antibody, preventive $\mathrm{Rh}$-typing match transfusion is estimated to effectively reduce up to $62 \%$ of the incidence of additional antibody in all allo-immunized patients. The occurrence of additional antibody can be almost completely inhibited if the combination of $\mathrm{S}$ and $\mathrm{Jk}^{\mathrm{b}}$ antibodies is considered. In addition, this observation agrees with the fact that antibody against the Rh system, along with antibodies against the Kidd and MNS systems, is the major type of antibodies against multiple systems.

Owing to the restrictions by the operation procedure, for immunized patients who already have antibody identification reports, the BRCBC performed antibody identification again only if unmatched blood was observed in the following cross-matching tests. Antibody screening was executed by medical institutes, and the results of pre-transfusion blood test were sent back to the BRCBC, so underestimation is possible. Nevertheless, to our knowledge and experience, despite the risk of appearance of a new antibody, we ensure that the blood samples match with the existing antibody present, which ensure the safety of blood transfusion. Another limitation is that our dataset cannot provide information about patients' history of blood transfusion and pregnancy before the occurrence of antibody. Therefore, we could not differentiate how many antibodies resulted from natural immune response or blood transfusion-induced immune response. This study did not evaluate the effects of different RBC blood components on the cumulative incidence of the same antibody type. However, previous studies showed that the effects of leukocyte and regular RBC products on the occurrence of antibody have no significant difference ${ }^{[22]}$. As the observation of cumulative incidence of additional antibody among the patients with the same type of immune disease in our study differed from that in the study that focused on European ethnic groups, a longer observation time is necessary so as to draw more-comprehensive conclusions. Therefore, the cumulative incidence of unexpected antibody after blood transfusion in in China still needs to be verified.

Among all the types of alloantibodies, anti-Rh was observed to have the highest prevalence (more than $60 \%$ of the alloantibody), followed by anti-MNS (including $14.4 \%$ anti-Mia), anti-Lewis, anti-Kidd, anti-Pl, and anti-Duffy. Anti-Rh accounts for $62.0 \%$ of the cases of additional antibodies among immunized patients. Performing Rh-compatible blood type preventive transfusion can significantly reduce nearly $60 \%$ of the unexpected antibodies. For example, the continuous increase in MNS blood type can further reduce the prevalence of unexpected antibody by $25 \%$. This applies to the inhibition of the occurrence of alloantibody and prevention of the increase in additional antibody. Calculations showed that the cumulative incidence of additional antibody among immunized patients was $5 \%$ at the first time, $1.82 \%$ at the second time, $2.33 \%$ at the fourth time, $3.07 \%$ at the fifth time, and $4.24 \%$ at the seventh time. In addition, the cumulative incidence of additional antibody among females was significantly higher than that among males. Therefore, the drive to perform preventive blood transfusion for senior people and females is stronger.

\section{Acknowledgments}

We thanks to partners from BRCBC for their mental support and staffs work in Blood Bank at Beijing for their material support.

\section{References}

[1] Sood R, Makroo RN, Riana V, et al. Detection of alloimmunization to ensure safer transfusion practice. Asian J Transfus Sci, 2013;7(2):135-9.

[2] Zalpuri S, Schonewille H, Middelburg R, et al. Effect of storage of red blood cells on alloimmunization. Transfusion, 2013;53(11):2795-800.

[3] Schonewille H, Doxiadis, II, Levering WH, et al. HLADRB1 associations in individuals with single and multiple clinically relevant red blood cell antibodies. Transfusion, 2014;54(8):1971-80.

[4] Chow MP, Hu HY, Lyou JY, et al. Red cells, HLA and platelet antibody formation in patients with multiple transfusions. Acta haematologica, 1994;92(2):57-60.

[5] Broadberry RE, Lin M. The incidence and significance of anti-"Mia" in Taiwan. Transfusion, 1994;34:349-52.

[6] Prathiba R, Lopez CG, Usin FM, et al. The prevalence of GP Mur and anti-"Mia" in a tertiary hospital in Peninsula Malaysia. Malays J Pathol, 2002;24(2):95-8. 
[7] Cheng CK, Lee CK, Lin CK, et al. Clinically significant red blood cell antibodies in chronically transfused patients: a survey of Chinese thalassemia major patients and literature review. Transfusion, 2012;52(10):2220-4.

[8] Wang Q, Yang Q, Bai Y, et al. Frequency of RBC Alloantibodies in Chinese Surgical Patients.Transfus Med Hemother, 2012;39(4):283-6.

[9] Mo Z, Li H, Huang L, et al. Prevalence and specificity of RBC alloantibodies in the general hospitalised population in Guangxi. Transfus Med, 2015;25(5):313-9.

[10] Xu P, Li Y, Yu H, et al. Prevalence, specificity and risk of red blood cell alloantibodies among hospitalised Hubei Han Chinese patients. Blood transfusion, 2014;12:56-60.

[11] Au WY, Liu CL, Lo CM, et al. Red blood cell alloantibodies and liver transplantation in Chinese patients. Transplantation, 2003;76(2):324-6.

[12] Wang Q, Yang Q, Bai Y, et al. WITHDRAWN: The frequency of RBC alloantibodies in Chinese surgical patients. Transfusion and apheresis science : official journal of the World Apheresis Association : official journal of the European Society for Haemapheresis, 2012.

[13] Schonewille H, de Vries RR, Brand A. Alloimmune response after additional red blood cell antigen challenge in immunized hematooncology patients. Transfusion, 2009;49(3):453-7.

[14] Schonewille H, van de Watering LM, Loomans DS, et al. Red blood cell alloantibodies after transfusion: factors influencing incidence and specificity. Transfusion, 2006;46(2):250-6.

[15] Sanz C, Nomdedeu M, Belkaid M, et al. Red blood cell alloimmunization in transfused patients with myelodysplastic syndrome or chronic myelomonocytic leukemia. Transfusion, 2013;53:710-5.

[16] Nordvall M, Dziegiel M, Hegaard HK, et al. Red blood cell antibodies in pregnancy and their clinical consequences: synergistic effects of multiple specificities. Transfusion, 2009;49(10):2070-5.

[17] Zalpuri S, Zwaginga JJ, le Cessie S, et al. Red-bloodcell alloimmunization and number of red-blood-cell transfusions. Vox Sang, 2012;102(2):144-9.

[18] Schonewille H, van de Watering LM, Brand A, et al. Additional red blood cell alloantibodies after blood transfusions in a nonhematologic alloimmunized patient cohort: is it time to take precautionary measures? Transfusion, 2006;46(4):630-5.

[19] Promwong C, Siammai S, Hassarin S, et al. Frequencies and specificities of red cell alloantibodies in the Southern Thai population. Asian J Transfus Sci, 2013;7(1):16-20.

[20] Zhu Z, Zeyan SW, Cheng Hoping. Rare blood group screening among Chinese blood donors in Shanghai area. Chin J Blood Transfusion, 2002;15:232-3.

[21] Wei CT, Al-Hassan FM, Naim N, et al. Prevalence of Diego blood group antigen and the antibody in three ethnic population groups in Klang valley of Malaysia. Asian J Transfus Sci, 2013;7(1):26-8.

[22] Schonewille H, Brand A. Alloimmunization to red blood cell antigens after universal leucodepletion. A regional multicentre retrospective study. Br J Haematol, 2005;129(1):151-6.

(Received 15 October 2016, Revised 28 November 2016, Accepted 10 December 2016) 